\title{
Die Wiener Gerichtsorganisation und die Organisation der Zivilgerichtsbarkeit seit 1848/50
}

\author{
The Organisation of Courts in Vienna and the Civil Courts System since 1848/50 \\ The present organisation of courts with its main characteristics was created, based on earlier structures, in the years \\ 1848 to 1854. Since then, many things have changed. It was not until 1898 that a new, modern civil procedure code \\ was enacted together with additional, modern accompanying legislation.
}

Keywords: civil justice law - organization of justice - Vienna courts

\section{Der Zeitraum von 1848 bis 1851}

Eine der Folgen der Revolution des Jahres 1848 war die Notwendigkeit der durchgehenden Verstaatlichung der Gerichtsbarkeit in erster Instanz (die bislang nur zum Teil durch staatliche Gerichte besorgt worden war) und in Verbindung damit die Schaffung einer neuen Gerichtsorganisation. ${ }^{1}$

Am Vorabend der Revolution gab es in Wien die Oberste Justizstelle (als Oberstes Gericht und oberste Administrativbehörde in Justizsachen), sodann, ihr nachgeordnet, ${ }^{2}$ das Niederösterrei-

\footnotetext{
${ }^{1}$ Der Blickwinkel dieses Aufsatzes liegt auf Wien einerseits im heutigen, andererseits im früher jeweils aktuellen Umfang, wobei es zum Verständnis der Organisationsvorschriften mitunter auch geboten erscheint, die Betrachtung auf ganz Niederösterreich auszudehnen. Die damals und seither getroffenen Maßnahmen sind, insbesondere was die zahlreichen Änderungen anlangt, komplex und vielschichtig, sodass in diesem Aufsatz auf deren Details und Hintergründe nicht näher eingegangen werden kann. Diese möge der geneigte Leser dem Werk des Verfassers "Staatliche Gerichte in Wien seit Maria Theresia" (m.w.N.) entnehmen - dessen erster Teil bezieht sich auf die Zeit bis 1848, der zweite auf die Zeit danach. ${ }^{2}$ Eine Struktur, die vor allem durch die Josefinischen Justizreformen geschaffen wurde.
}

chische Appellationsgericht als Gericht zweiter Instanz (auch für Oberösterreich und Salzburg zuständig), und weiters eine bunte Landschaft an Gerichten erster Instanz: Hervorzuheben sind das Nö. Landrecht, ein reines Zivilgericht (vor allem der Personalgerichtsstand ${ }^{3}$ des Adels, aber auch mit weiteren Kompetenzen), dann das (mit dem Nö. Landrecht verknüpfte) Merkantil- und Wechselgericht; der Magistrat der Stadt Wien mit dem politisch-ökonomischen Senat, ${ }^{4}$ dem Zivilsenat (1841: Zivilgericht), und dem Kriminalsenat (1841: Kriminalgericht), beide Letztere jeweils unter der Leitung eines Vizebürgermeisters. Ergänzend gab es im politischökonomischen Senat eine Senatsabteilung für die Schweren Polizeiübertretungen. ${ }^{5}$ Schließlich

\footnotetext{
${ }^{3}$ Der Personalgerichtsstand ist ein Vorläufer dessen, was wir heute als allgemeinen Gerichtsstand bezeichnen (Gegensatz: insbesondere der Realgerichtsstand). ${ }^{4}$ Das ist der Magistrat im heutigen (engeren) Sinn.

${ }^{5}$ Das Strafgesetzbuch (StB) 1803 behandelte in seinem I. Teil die Verbrechen (samt Prozessrecht) und in seinem II. die Schweren Polizeiübertretungen (das sind in gewissem Sinne die Vorläufer der heutigen Vergehen), ebenfalls samt dem Prozessrecht - das war eine Art "gerichtliches Verwaltungsstrafrecht" mit dem Rechtszug an die politischen (= Verwaltungs-) Behörden.
} 
gab es zahlreiche Patrimonialgerichte (Gerichte der Grundherrschaften) unterschiedlicher GröBe. ${ }^{6}$

Noch im Frühjahr 1848 wurde die Oberste Justizstelle in den Obersten Gerichtshof und das Justizministerium aufgespalten. ${ }^{7}$

Die eingehenden weiteren Planungen brachten für Niederösterreich (samt Wien) folgende neue Gerichtsorganisation:8

Das Nö. Appellationsgericht wurde in zwei Oberlandesgerichte geteilt: Eines für Niederösterreich (in Wien), das andere für Oberösterreich und Salzburg in Linz. ${ }^{9}$

Weiters wurden vier Landesgerichte errichtet: In Wien (auch für den Sprengel des BG Tulln und den größten Teil des Weinviertels, einen Gerichtshof in Korneuburg gab es damals noch nicht), Krems, St. Pölten und Wiener Neustadt, dazu ein Handelsgericht für den Sprengel des Landesgerichtes Wien (ansonsten waren die Handelssenate der Landesgerichte zuständig).

Schließlich wurden (außerhalb von Wien) 73 Bezirksgerichte errichtet, davon waren elf "Bezirkskollegialgerichte“, die übrigen 62 (dazu kamen acht weitere in Wien) „einfache“ Bezirksgerichte (Einzelrichter). Die „Schweren

\footnotetext{
${ }^{6}$ Außer Betracht bleiben hier das Obersthofmarschallamt, die Militärgerichte (sie übten eine vollständige Gerichtsbarkeit aus, in Zivilsachen bis 1869, in Strafsachen bis 1920), die Gefällsgerichte (in gewissen Steuerangelegenheiten), sowie die Gerichtsbarkeit des Öffentlichen Rechts (insbesondere Reichsgericht / Verfassungsgerichtshof, Verwaltungsgerichtshof, Bundesgerichtshof).

7 „Vorschrift" des Justizministers vom 15. 5. 1848; das in der Justizgesetzsammlung (JGS), Nr. 1176, abgedruckte Justizhofdekret vom 31. 8. 1848 war bloß eine (neu datierte) nachträgliche Bekanntmachung. Zur Entstehungsgeschichte siehe WALDSTÄTTEN, Gerichte, 78, Anm. 279, unter Hinweis auf Josef KASERER, Handbuch der Österreichischen Justizverwaltung, 1. Bd., 20.

${ }^{8}$ RGBl. 288/1849; zum Werdegang siehe ausführlich bei KoHL, Gerichtsorganisation.

${ }^{9}$ RGB1. 289/1849.
}

Polizeiübertretungen" (StG 1803) wurden in Vergehen einerseits (das waren die schwereren Delikte) und Übertretungen andererseits (das waren die minderen Delikte) unterteilt. Die Bezirkskollegialgerichte waren zuständig als Erkenntnisgerichte für Vergehen und als Untersuchungsgerichte für Verbrechen (überdies hatten die Landesgerichte für einen engeren Sprengel auch die Funktion eines Bezirkskollegialgerichtes); die Übertretungen gehörten vor die Bezirksgerichte schlechthin. Die Landesgerichte (als solche) waren (insbesondere) zuständig als Erkenntnisgerichte für Verbrechen.

In Wien (im damaligen Umfang) wurden acht Bezirksgerichte entsprechend den (nach Eingemeindung der so genannten Vorstädte) damals vorgesehenen acht Stadtbezirken errichtet (der spätere Bezirk Margareten war noch Teil von Wieden). ${ }^{10}$ Das BG Innere Stadt hatte wegen seiner Bedeutung drei Sektionen, wobei die dritte als Landtafel- und (für das Gebiet aller acht Wiener BGe) Grundbuchsamt fungierte. ${ }^{11}$ Von den weiteren Bezirksgerichten in Niederösterreich hatten nur drei ihren Sitz im heutigen

\footnotetext{
${ }^{10}$ Mehrfach liest man, dass Wien damals „bis zum Linienwall“ (der ungefähr dort verlief, wo später die Gürtelstraße errichtet wurde) ausgeweitet wurde. Das trifft nur für den Bereich nördlich von Gaudenzdorf zu. Südlich davon reichte die neue (sehr unregelmäßig verlaufende) Stadtgrenze bis zum St. Marxer Friedhof zum Teil weit über den Linienwall hinaus, was von geringer praktischer Bedeutung war, weil dieses Gebiet weitgehend unbebaut war. Zum ungefähren Verlauf siehe WALDSTÄTTEN, Gerichte, 89f., insb. Anm. 323. Der 1874 neu gebildete Stadtbezirk Favoriten (damals viel kleiner als heute) wurde ohne Stadterweiterung auf Stadtgebiet errichtet. Der Bezirk Margareten wurde erst 1861/62 gebildet, die neue Bezirkseinteilung wurde 1862 effektiv.

${ }^{11}$ Die beiden anderen Sektionen waren jeweils für einen bestimmten Teil der Inneren Stadt zuständig. Beschreibung der Sprengel der Wiener BGe: nö. LGBl. 77/1849.
} 
Stadtgebiet, nämlich Hietzing, Sechshaus und Hernals. ${ }^{12}$

In Niederösterreich wurden (außerhalb der Stadt Wien) 17 Bezirkshauptmannschaften errichtet. ${ }^{13}$

Parallel zu diesen Organisierungsarbeiten wurde gleichsam ein ganzes Paket an neuen Normen geschaffen: Die materiellen Teile des StG 1803 wurden etwas modifiziert, ${ }^{14}$ die prozessualen Teile wurden durch eine neue (liberale) Strafprozessordnung ersetzt, ${ }^{15}$ es wurden eine neue Jurisdiktionsnorm, ${ }^{16}$ ein Außerstreitgeset $^{17}$ und ein Organisches Gesetz über die Gerichtsstellen $^{18}$ geschaffen. Die Allgemeine gerichtsordnung (AGO) war weiterhin anzuwenden.

Das neue Oberlandesgericht in Wien wurde zum 1. Mai 1850 aktiviert, $^{19}$ der Beginn der Wirksamkeit der übrigen neuen Gerichtsbehörden (einschließlich der Staatsanwaltschaften) für Niederösterreich (samt Wien) erfolgte mit dem 1. Juli $1850 .{ }^{20}$ Die Umsetzung erfolgte sichtlich klaglos.

Mit Patent vom 7. August 1850 wurde das Statut des Obersten Gerichts- und Kassationshofes erlassen..$^{21}$ Das oberste Gericht war nun für das gesamte Gebiet der Monarchie, auch für Ungarn, zuständig; der bisher bestandene lombardo-venetianische Senat wurde aufgehoben. ${ }^{22}$

\footnotetext{
${ }^{12}$ Wobei das heutige Stadtgebiet auch zu den Sprengeln anderer Bezirksgerichte gehörte. Siehe den Plan in Opll, Karten, Tafel 48, wiedergegeben auch in WALDSTÄTtEN, Gerichte, 428 und 429.

${ }^{13}$ Nö. LGB1. 353/1849.

${ }^{14}$ U.a. RGBl. 24/1850.

${ }^{15}$ RGBl. 25/1850.

${ }^{16}$ RGBl. 237/1850.

${ }^{17}$ RGB1. 255/1850.

${ }^{18}$ RGBl. 258/1850.

${ }^{19}$ RGBl. 138/1850.

${ }^{20}$ RGBl. 234/1850.

${ }^{21}$ RGB1. 325/1850.

${ }^{22}$ Bildung eines Aushilfssenates beim OGH für die ungarischen Angelegenheiten: Wiener Zeitung 24. 12. 1850, 3912.
}

Ergänzend erging eine Notariatsordnung; ${ }^{23}$ ein Grundbuchsgesetz wurde zwar angekündigt, kam aber erst 1871 zustande.

\section{Der Zeitraum von 1852 bis 1867}

Mit den beiden kaiserlichen Patenten vom 31. Dezember $1851,^{24}$ bekannt als "Silvesterpatente", erfolgte auch formell der Übergang zum Neoabsolutismus. Mit kaiserlichem Kabinettschreiben vom selben $\mathrm{Tag}^{25}$ wurden die Grundsätze für die Neuordnung des Reiches bekannt gegeben. Soweit hier von Bedeutung, waren u.a. zwei Gesichtspunkte besonders erheblich: Die neue Organisation hatte billiger zu sein und vor allem waren außerhalb der Städte mit Gerichtshöfen Bezirksämter zu errichten, die für Administrativ-, Justiz- und Fiskalangelegenheiten zuständig sein sollten (so genannte "gemischte Bezirksämter").26

Umgehend wurden die Geschworenengerichtsbarkeit und die Generalprokuratur beim Obersten Gerichtshof aufgehoben. ${ }^{27}$ Mit 1. September 1852 trat ein neues StG in Kraft, das, wenngleich vielfach novelliert, bis Ende 1974 galt. ${ }^{28}$

${ }^{23}$ RGBl. 366/1850.
${ }^{24}$ RGBl. 2 und 3/1852.

${ }^{25}$ RGBl. 4/1852.

${ }^{26}$ Das ist eine verkürzte Darstellung. Siehe näher insbesondere bei KOHL, Gerichtsorganisation, m.w.N. Motiv für die Vereinigung der Geschäfte bei einer (einzigen) Behörde sollen Klagen der Bevölkerung über zu große Sprengel bzw. leichtere Erreichbarkeit der Behörden gewesen sein (Ebd. 41). Die Wünsche waren wohl nicht unberechtigt, bedenkt man, dass an die Stelle der sehr zahlreichen Patrimonialbehörden viel weniger staatliche Behörden getreten waren; dem Grundsatz der Trennung von Justiz und Verwaltung kam damals gegenüber solchen praktischen Überlegungen sichtlich kein relevanter Stellenwert zu.

${ }^{27}$ RGBl. 5, 24/1852.

${ }^{28}$ RGBl. 117/1852; das war eine ergänzte, aber auch überarbeitete und auch neu gefasste Ausgabe der materiellen Teile des StG 1803. Begleitend dazu die 
Die Schaffung einer neuen Gerichtsorganisation nahm einige Zeit in Anspruch, wobei auch neue Gesetze ausgearbeitet wurden (die erst mit der Wirksamkeit der neuen Gerichtsorganisation in Kraft traten), so die Zivil-Jurisdiktionsnorm,29 eine Strafprozessordnung, ${ }^{30}$ wie auch ein Außerstreitgesetz (es galt, ebenfalls vielfach novelliert, bis Ende 2004). ${ }^{31}$

Nach der neuen Organisation ${ }^{32}$ waren für Niederösterreich (samt Wien) vorgesehen:

a) Ein Oberlandesgericht, nun wieder zuständig auch für Oberösterreich und Salzburg.

b) Sechs Gerichtshöfe erster Instanz, nämlich das Landesgericht sowie das Handelsgericht in Wien (für den Sprengel des LG), dann Kreisgerichte $^{33}$ in Korneuburg (womit der Sprengel des LG Wien und des HG Wien nicht mehr in das Weinviertel reichte), ${ }^{34}$ Krems, St. Pölten und Wiener Neustadt.

c) Außerhalb der Orte der Gerichtshöfe erster Instanz „gemischte Bezirksämter", von denen manche als Untersuchungsgerichte bestimmt waren - die früheren Bezirkskollegialgerichte

Verordnung RGB1. 118/1852 zur Anpassung der Zuständigkeiten.

${ }^{29}$ RGBl. 251/1852.

${ }^{30}$ RGB1. 151/1853; sie war nicht liberal wie ihre Vorgängerin ex 1850, sondern brachte wiederum den Inquisitionsprozess in veränderter Form.

${ }^{31}$ RGBl. 208/1854.

${ }^{32}$ RGBl. 249/1853.

33 Wohl aus der Überlegung, dass bei den Kreisgerichten (typologisch) weniger wichtige Sachen anfielen als beim Landesgericht, waren die Richter der KGe schlechter besoldet als jene des LG. Das KG hatte auch keinen Präsidenten, sondern einen Präses Angleichung erst 1869, RGBl. 48.

${ }^{34}$ Südlich der Donau umfasste der Sprengel des LG Wien nicht mehr jenen des Bezirksamtes (BA) Tulln, wohl aber weiterhin jene der BAe Bruck an der Leitha, Hainburg, Schwechat, Mödling, Hietzing, Sechshaus, Hernals, Purkersdorf und Klosterneuburg. Die Ausweitung des LG-Sprengels auf Gebiete nördlich der Donau erfolgte erst zu Beginn des 20. Jhdts. gab es nicht mehr. ${ }^{35}$ Die Bezirkshauptmannschaften wurden aufgelöst (im Prinzip entsprachen die Sprengel der Bezirksämter mit gewissen Abweichungen jenen der bisherigen Bezirksgerichte).

d) An den Orten der Gerichtshöfe erster Instanz städtisch-delegierte Bezirksgerichte. Das waren keine selbständigen Bezirksgerichte mehr, sondern gewissermaßen Exposituren der Gerichtshöfe. ${ }^{36}$ Sie waren (weiterhin) in Zivil- wie auch in Strafsachen zuständig, hatten aber geringere Kompetenzen als die Bezirksämter, ${ }^{37}$ insbesondere kam die Grundbuchsführung dem Gerichtshof zu (bis 194038).

Was Wien anlangt, wurden die bisherigen acht Bezirksgerichte in städtisch-delegierte Bezirksgerichte umgewandelt (die dritte Sektion des BG Innere Stadt, das Landtafel- und Grundbuchsamt, kam zum Landesgericht selbst).

Das neu gebildete Oberlandesgericht wurde mit 31. August 1854 aktiviert, ${ }^{39}$ die übrigen Gerichtsbehörden und Bezirksämter mit 30. September 1854, zugleich hatten die verschiedenen Vorschriften, die auf die neue Organisation abstellten, in Kraft zu treten ${ }^{40}$.

Diese Organisation blieb nun längere Zeit stabil. ${ }^{41}$

Das Landesgericht in Wien war (weiterhin) ein einheitliches Gericht. ${ }^{42} 1859$ wurde erwogen, es

\footnotetext{
${ }^{35}$ Als Erkenntnisgerichte hinsichtlich der Vergehen hatten die Gerichtshöfe erster Instanz einzuschreiten.

${ }^{36}$ Sie hatten kein eigenes Personal, es gehörte zum Gerichtshof - ein Zustand, der bei den Richtern bis zum Richterdienstgesetz 1962 andauerte. An den Orten der Kreisgerichte wurden demnach „politische Bezirksämter" errichtet; Wien behielt seine Sonderstellung.

${ }^{37}$ Siehe dazu näher KOHL, Gerichtsorganisation, insbesondere 108 .

38 dRGB1. I 301.

${ }^{39}$ RGBl. 206/1854.

${ }^{40}$ RGBl. 216, 218/1854.

${ }^{41} \mathrm{Im}$ Kern haben wir heute noch die (wenngleich vielfach geänderte) Gerichtsorganisation 1854 .
} 
wegen seiner Größe in ein LG für Zivil- und eines für Strafsachen zu teilen. Das erfolgte zwar nicht, doch wurde dem Leiter der strafgerichtlichen Abteilung hinsichtlich seines Bereiches ein gewisses Maß an Selbständigkeit eingeräumt. ${ }^{43}$

Der für die Monarchie unglücklich verlaufene Krieg 1859 brachte allmählich die Rückkehr zum Konstitutionalismus. Aufgrund des Oktoberdiplomes vom 20. Oktober 1860 erlangte, soweit hier erheblich, Ungarn wieder seine frühere relative Selbständigkeit, was zur Folge hatte, dass der Wirkungsbereich des Justizministeriums wie auch des Obersten Gerichtshofes entsprechend eingeschränkt wurde. ${ }^{44}$

1863 wurden das Allgemeine Handelsgesetzbuch (AHGB) in Kraft gesetzt ${ }^{45}$ und ein neues Handelsregister eingeführt. ${ }^{46}$

Der ebenfalls verlorene Krieg 1866 brachte die Dezemberverfassung 1867.

\section{Der Zeitraum von 1868 bis 1918}

Die neuen staatsrechtlichen Verhältnisse brachten (abermals und endgültig) die Trennung von Justiz und Verwaltung: Mit 31. August 1868

\footnotetext{
${ }^{42}$ Es war aber von Anfang an (Juli 1850) disloziert: Das Präsidium und die Zivilabteilung befanden sich in einem heute nicht mehr bestehenden Gebäude am Minoritenplatz (sie übersiedelten sodann 1881 in den Justizpalast), die Strafabteilung im „Grauen Haus“. Es gab einen Präsidenten und 1850 drei „Senatspräsidenten“, ab der Reform 1854 drei Vizepräsidenten. Der Präsident (mit einem Vizepräsidenten) leitete das gesamte Gericht und die Zivilabteilung, ein Vizepräsident - wohl der rangälteste - (mit dem weiteren) die Strafabteilung; dieser erhielt 1873 den Titel „Zweiter Präsident“ (der Präsident des LG den Titel „Erster Präsident").

${ }^{43}$ In diesem Sinne später und mit anderem Wortlaut für andere Landesgerichte Justizamtsblatt 10/1905.

${ }^{44}$ Siehe RGBl. 225, 226/1860 und die in der Wiener Zeitung vom 21.10. 1860 wiedergegebenen kaiserlichen Handschreiben.

${ }^{45}$ RGBl. 1/1863.

${ }^{46}$ RGBl. 27/1863.
}

traten, soweit hier erheblich, in Niederösterreich an die Stelle der gemischten Bezirksämter Bezirksgerichte und 18 Bezirkshauptmannschaften. ${ }^{47}$ Die städtisch-delegierten Bezirksgerichte blieben bestehen.

1871 wurde ein Grundbuchsgesetz erlassen; ${ }^{48}$ die weiteren Gesetze zur Anlage der Grundbücher ergingen in der Folge, für Niederösterreich (samt Wien) 1874. ${ }^{49}$

"Dauerthemen" der damaligen Jahre waren die Schaffung einer modernen Strafprozessordnung wie auch einer modernen Zivilprozessordnung (an Stelle der Allgemeinen Gerichtsordnung). Ersteres gelang: Die neue StPO trat am 1. Jänner 1874 in Kraft. ${ }^{50}$ Zweiteres gelang vorerst nicht; allerdings ergingen das Gesetz über das Mahnverfahren ${ }^{51}$ und das Gesetz über das Bagatellverfahren, ${ }^{52}$ das in gewissen geringfügigen Streitsachen ein vereinfachtes Verfahren (nach modernen Grundsätzen) vor den Bezirksgerichten brachte (und dem entsprechend auch die Errichtung des Bagatellgerichtes für Handelssachen in Wien $\left.^{53}\right)$.

Das Anwachsen der Bevölkerung in Wien und in den Vororten hatte naturgemäß auch eine starke Steigerung der Arbeit bei den Gerichten zur Folge. Das führte zum Entschluss, überlastete Bezirksgerichte $\mathrm{zu}$ teilen. ${ }^{54}$ Vom BG Hernals

${ }^{47}$ RGBl. 44, 101, 117/1868.

${ }^{48}$ RGB1. 95/1871.

${ }^{49}$ RGBl. 88/1874; die Durchführung der Anlage der neuen Grundbücher (das sind diejenigen, die durch das EDV-Grundbuch abgelöst wurden) nahm Jahre in Anspruch und war in Wien im ersten Quartal 1885, im übrigen Niederösterreich im Jahr 1890 beendet (Justizamtsblatt 1885, 110; 1890, 46).

${ }^{50}$ RGBl. 119/1873; damit wurde auch die Generalprokuratur beim Obersten Gerichtshof wieder errichtet. Diese StPO gilt, oftmals auch wesentlich geändert und wiederverlautbart, noch heute.

${ }^{51}$ RGBl. 66/1873.

52 RGBl. 67/1873.

${ }^{53}$ RGBl. 101/1873.

${ }^{54} \mathrm{Zu}$ große Einheiten waren nach den damaligen Auffassungen unerwünscht, zumal es auch Probleme 
wurden die BGe Ottakring und Währing abgespalten $^{55}$ (aktiviert mit 18. Juni 1877 bzw. 15. Jänner 187756). Das BG Wieden wurde geteilt und es wurden die BGe Margareten und Favoriten errichtet ${ }^{57}$ (Aktivierung beider Gerichte per 1. November $\left.1882^{58}\right) .{ }^{59}$ Ebenfalls geteilt wurde das BG Sechshaus, ${ }^{60}$ es entstanden die BGe Fünfhaus (per 1. Juni 188861) und Untermeidling (später: Meidling; aktiviert mit 1. Jänner 188562). Es folgten die Teilung des BG Innere Stadt ${ }^{63}$ in Innere Stadt I (das ist das "alte“) und II (das ist das "neue“, aktiviert mit 1. Jänner $\left.1890^{64}\right)$, sodann 1890 die Teilung des BG Leopoldstad ${ }^{65}$ in Leopoldstadt I (das ist das „alte“) und II (das ist das „neue“, ohne Strafsachen; aktiviert zum 1. August 189166).

Sozusagen parallel dazu setzte die Entwicklung ein, bezirksgerichtliche Strafsachen sukzessive bei bestimmten Bezirksgerichten zu konzentrieren.67 Das BG Alsergrund übernahm mit

mit der Unterbringung gab. Überhaupt war Raumnot oft ein Grund für Organisationsänderungen. Nach der damaligen Gesetzestechnik wurde das neue Gericht zunächst durch Verordnung (grundsätzlich) errichtet und sodann mit weiterer Verordnung aktiviert. Oben werden die Daten der Aktivierung genannt.

${ }^{55}$ RGBl. 24, 25/1876.

${ }^{56}$ RGBl. 24, 130/1876.

${ }^{57}$ RGBl. 13/1882.

${ }^{58}$ RGBl. 63/1882.

${ }^{59}$ Das BG Favoriten war zunächst nur in Zivilsachen zuständig, ab 1891 (RGBl.60) auch in Strafsachen (Grundbuchsführung ab 1893 - zuvor beim Landesgericht).

${ }^{60}$ RGBl. 176/1882.

${ }^{61}$ RGB1. 25/1888.

62 RGBl. 164/1884.

${ }^{63}$ RGBl. 132/1889.

${ }^{64}$ RGB1. 154/1889.

${ }^{65}$ RGBl. 192/1890.

${ }^{66}$ RGBl. 75/1891.

${ }^{67}$ Das hatte vor allem praktische Gründe, wie insbesondere Raumnot, und dauerte Jahrzehnte. Abschluss der Entwicklung waren die Strafabteilungen des Amtsgerichtes Wien, aus denen 1945 das StrafBG Wien entstand. Das BG Alsergrund hatte seinen Sitz im „Grauen Haus“.
1. Jänner 1880 die Strafsachen vom BG Josefstadt ${ }^{68}$ und mit 1. Mai 1881 vom BG Innere Stadt. ${ }^{69}$

Anfang der 1890er-Jahre wurde Wien durch die Eingemeindung der Vororte wesentlich erweitert und zählte nun 19 Bezirke (alle südlich der Donau). ${ }^{70}$ Dazu wurde die Wiener Gerichtsorganisation den neuen Gegebenheiten angepasst (per 1. April 189271): Die einbezogenen Bezirksgerichte und das neu errichtete BG Simmering wurden zu städtisch-delegierten Bezirksgerichten (Untermeidling wurde in Meidling, Sechshaus in Rudolfsheim umbenannt), hatten aber (ebenso wie das BG Favoriten) die Gerichtsbarkeit für die außerhalb des Standortes eines Gerichtshofes geltenden Bestimmungen auszuüben. Dazu kam es zu Anpassungen der Sprengel entsprechend der neuen Bezirkseinteilung.

Im zeitlichen Zusammenhang mit der Stadterweiterung wurde das BG Döbling errichtet (abgetrennt vom BG Währing, per 1. Jänner 189472).

Nach jahrelangen Vorarbeiten trat mit 1. Jänner 1898 die große Reform der Zivilverfahrensgesetze (samt weiteren Normen) in Kraft:73 Die Zivilprozessordnung (ZPO), Jurisdiktionsnorm (JN), Exekutionsordnung (EO), dann auch ein neues Gerichtsorganisationsgesetz (GOG) und eine neue Geschäftsordnung (Geo).

Auf die Wiener Gerichtsorganisation selbst hatte die Reform relativ geringe Auswirkungen. ${ }^{74}$ Das

${ }^{68}$ RGBI. 115/1879.

${ }^{69}$ RGBl. 2/1881.

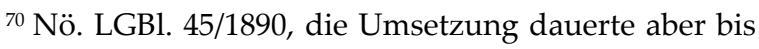
1892. S. näher WALDSTÄTTEN, Gerichte, 183f, m.w.N. Der 20. Bezirk entstand (durch Teilung des 2.) erst 1900 (nö. LGBl. 17).

${ }^{71}$ RGBl. 36/1892.

72 RGBl. 154/1893.

${ }^{73}$ Gesetze RGBl. 110-113/1895, RGBl. 78, 79, 217/1896, RGB1. 112/1897.

${ }^{74}$ Wohl aber gab es einen vermehrten Personal- wie auch Raumbedarf (Verhandlungssäle), was auch zu Übersiedlungen führte. S. ebenfalls WALDSTÄTTEN, Gerichte, 190-193. Die Frage des vermehrten Personalbedarfes wäre näher zu untersuchen: Das Verfah- 
Landesgericht Wien blieb ein einheitliches Gericht, die beiden Abteilungen erhielten aber die Bezeichnung Landesgericht in (nicht: für) Zivilrechtssachen und Landesgericht in Strafsachen, die Leiter den Titel „Präsident“ (des ...). Der Unterschied zwischen den städtisch-delegierten und den übrigen Bezirksgerichten wurde (grundsätzlich) beseitigt, ${ }^{75}$ die Bezeichnung „städtisch-delegiert" verschwand. Neu errichtet wurde das Exekutionsgericht Wien (formell als Expositur des BG Innere Stadt $\left.\mathrm{II}^{76}\right)$; vereinigt wurden die BGe Mariahilf und Neubau (zum BG Neubau), dann die BGe Josefstadt und Alsergrund, Letztere aber zugleich in die BGe Josefstadt in Zivilsachen und Josefstadt in Strafsachen geteilt. ${ }^{77}$

Zum 1. Jänner 1898 wurden übrigens auch die noch heute gebräuchlichen Talare eingeführt (samt Baretten: "Amtskleid“) ${ }^{78}$ - zuvor wurde in Uniform verhandelt (es gab eine eigene Uniform für die Beamten, zu denen auch die Richter und Staatsanwälte zählten).

Mit 1. Juli 1898 wurde in Wien das Gewerbegericht (Vorläufer des Arbeitsgerichtes) errichtet.79

ren nach der Allgemeinen Gerichtsordnung war zwar im Wesentlichen schriftlich, doch erfolgten auch damals die Einvernahmen zu Beweiszwecken mündlich. Und das nimmt heutzutage im Zivilprozess erster Instanz die allermeiste Zeit in Anspruch.

${ }^{75}$ Wenngleich weiterhin die Landes- und Kreisgerichte für ihren engeren Sprengel die Grundbücher zu führen hatten.

${ }^{76}$ RGBl. 157/1897.

${ }_{77}$ RGBl. 282/1897; dem BG Josefstadt in Strafsachen, zuständig für die Bezirke 1, 8 und 9, wurde mit 1. 5. 1899 auch die Strafgerichtsbarkeit für den 16. Bezirk übertragen - RGBl. 235/1898 (es gab auch spätere Änderungen). Dieses Gericht ist der Vorläufer des StrafBG.

${ }^{78}$ RGB1. 187/1897.

${ }^{79}$ RGBl. 58/1898; das Gesetz RGB1. 63/1869 hatte die Möglichkeit zur Errichtung von Gewerbegerichten geschaffen, das waren aber keine staatlichen, sondern reine Laiengerichte. Die „neuen“ Gewerbegerichte auf Grundlage des Gesetzes RGB1. 218/1896 waren staatliche Gerichte. 1946: „Arbeitsgerichte“ (BGBl. 170). Zu
Mit 1. Jänner 1904 wurde das (noch nicht in Wien gelegene) BG Liesing aktiviert. ${ }^{80}$

1905 wurden größere Gebiete auf der linken Donauseite als 21. Bezirk eingemeindet, 1910 dann noch der restliche Teil der Ortsgemeinde Strebersdorf. ${ }^{81}$ Diese Gebiete bildeten den Sprengel des BG Floridsdorf. ${ }^{82}$

Seit 1850/54 galt der Grundsatz (mit Ausnahmen), dass das Bezirksgericht durch einen Einzelrichter, der Gerichtshof erster Instanz in Zivilsachen durch einen Dreiersenat, das Oberlandesgericht durch einen Fünfer- und der Oberste Gerichtshof durch einen Siebenersenat entschieden. 1907 wurde dies insofern geändert, als nun der Oberste Gerichtshof grundsätzlich in Fünfersenaten und das Oberlandesgericht in bestimmten Fällen in Dreiersenaten zu entscheiden hatte (später wurde beim Oberlandesgericht der Dreiersenat die Regel). ${ }^{83}$

Noch vor Beginn des Ersten Weltkrieges kam es beim Gerichtshof erster Instanz zum Beginn des Einzelrichterverfahrens in Zivilsachen. ${ }^{84}$

1908 übersiedelten die beiden Bezirksgerichte Innere Stadt in den neu errichteten ersten Bauteil des Gerichtsgebäudes Riemergasse und wurden dort vereinigt. ${ }^{85}$ Mit 1. Jänner 1913 wurden die BGe Wieden und Margareten zum BG Margareten (mit Sitz im Gerichtsgebäude Mittersteig, dessen zweiter Bauteil fertig gestellt worden war) vereinigt. ${ }^{86}$ Der Neubau des Gerichtstraktes des BG Leopoldstadt I (Schiffamts-

den Schiedsgerichten der Sozialversicherungen s. WALDSTÄTTEN, Gerichte, $193 \mathrm{f}$.

${ }^{80}$ RGB1 220/1903; errichtet RGB1. 187/1901.

${ }^{81}$ Nö. LGBl. 1/1905, 170/1910.

82 RGBl. 208-210/1905; das BG Floridsdorf war 1895/96 im Sprengel des KG Korneuburg errichtet worden: RGB1. 160/1895, 138/1896, und kam nun zum Sprengel der Wiener Gerichtshöfe erster Instanz.

${ }^{83}$ RGBl. 41/1907.

${ }^{84}$ RGBl. 118/1914.

${ }^{85}$ RGBl. 43/1908; dorthin übersiedelten auch das Exekutionsgericht und die Auktionshalle.

${ }^{86}$ RGBl. 206/1912. 
gasse / Donaustraße) wurde 1915 abgeschlossen; beide BG Leopoldstadt wurden dort zum 1. August 1915 unter der Bezeichnung BG Leopoldstadt vereinigt. ${ }^{87}$

\section{Die Erste Republik (1918-1938)}

Noch 1918 wurde beim Gerichtshof (unter bestimmten Voraussetzungen) das als befristetes Provisorium gedachte Einzelrichterverfahren in Strafsachen („Vereinfachtes Verfahren“) eingeführt, $^{88}$ sodann 1920 die Schöffengerichtsbarkeit. ${ }^{89}$

Das Jahr 1920 brachte auch die Aufhebung der Militärgerichtsbarkeit per 1. Oktober $1920.9^{90}$ Damit stand der (,zivilen“) Justizverwaltung das große Militärgerichtsgebäude am Hernalser Gürtel zur Verfügung. Das (bislang einheitliche) Landesgericht Wien wurde zum 1. Oktober 1920 in drei selbständige Gerichtshöfe geteilt::11 In das Landesgericht für (nicht mehr: „in“) Zivilrechtssachen (ZRS), das Landesgericht für Strafsachen I (LGSt I) mit Sitz im "Grauen Haus" und das Landesgericht für Strafsachen II (LGSt II) mit Sitz im ehemaligen Militärgerichtsgebäude am Hernalser Gürtel. ${ }^{22}$ Der Sprengel des LGSt I umfasste die bevölkerungsreicheren Wiener Bezirksgerichts-Sprengel, der des LGSt II die übrigen und jene der außerhalb von Wien gelegenen. ${ }^{93} 1921$ gab es eine Anpassung der Spren-

\footnotetext{
${ }^{87}$ RGBl. 194/1915.

88 StGBl. 93/1918; bis 1926; abermals eingeführt mit der Novelle BGB1. I 82/1934.

${ }^{89}$ StGBl. 279/1920.

${ }^{90}$ StGBl. 321/1920.

91 StGBl. 402/1920.

92 Das Gebäude wird demnach im Volksmund immer noch das "Zweier-Landl“ genannt.

${ }^{93}$ Gemäß der Größe der Gebäude und der Kapazität der Gefangenenhäuser war das Verhältnis etwa 2: 1 (LG I zu LG II). Die Sprengeleinteilung änderte sich in der Folge etwas.
}

gel. ${ }^{94}$ Das BG Josefstadt in Strafsachen (dessen Zuständigkeiten ausgeweitet wurden) wurde in "Strafbezirksgericht I in Wien" umbenannt (weil es dem LGSt I unterstand ${ }^{95}$ ), das BG Josefstadt in Zivilsachen noch 1921 in (nur) BG Josefstadt.

Mit 15. Oktober 1920 wurde in Wien ein eigenes Jugendgericht als selbständiges Bezirksgericht geschaffen. ${ }^{96}$ Seine Zuständigkeiten wurden 1923/1925 erweitert,97 mit 1. Jänner 1929 entstand daraus der Jugendgerichtshof. ${ }^{98}$

Die Eingliederung des Burgenlandes in die Republik Österreich war für die Wiener Gerichtsorganisation insofern von Belang, als das Gebiet den Wiener Gerichtshöfen (nicht auch dem LGSt I, sondern nur dem LGSt II) unterstellt wurde. ${ }^{99}$

In der Zwischenkriegszeit kam es (vorwiegend aus Ersparungsgründen) zur Vereinigung einiger Wiener Bezirksgerichte: Favoriten und Simmering zum BG Favoriten mit 1. Oktober 1920;100 Rudolfsheim und Fünfhaus mit 15. Oktober 1922 zum BG Fünfhaus; ${ }^{101}$ Ottakring und Hernals mit 20. September 1924 zum BG Hernals; 102 Währing und Döbling mit 16. November 1925 zum BG Döbling. ${ }^{103}$ Im Herbst 1931 war der 1927 ausgebrannte Justizpalast soweit wieder hergestellt, dass er bezogen werden konnte. Es wurden dort die BGe Innere Stadt und Neubau un-

\footnotetext{
${ }^{94}$ BGBl. 310/1921.

${ }^{95}$ BGBl. 311/1921.

${ }^{96}$ StGBl. 439/1920 auf Grundlage des Gesetzes StGBl. 46/1919.

${ }^{97}$ BGBl. 119/1923, 24/1925.

98 BGBl. 234/1928.

${ }^{99}$ BGBl 476/1921, 18/1922; das Landesgericht Ödenburg konnte ja nicht errichtet werden. Der „Schattendorfer Prozess" (1927) fand somit vor dem LGSt II statt, allerdings im großen Schwurgerichtssaal des LGSt I (weil das LGSt II keinen so großen Saal hatte).

${ }^{100}$ StGB1. 429/1920.

101 BGBl. 665/1922.

102 BGBl. 334/1924.

${ }^{103}$ Justizamtsblatt Nr. 32.
} 
tergebracht und Letzteres per 1. Jänner 1932 mit dem BG Innere Stadt vereinigt. ${ }^{104}$

1926 wurde ein neues Gerichtsorgan geschaffen:

Der Rechtspfleger; ${ }^{105}$ die Einrichtung bewährte sich und der Wirkungskreis wurde erstmals $1929^{106}$ und sodann in weiterer Folge mehrfach ausgeweitet. ${ }^{107}$

\section{Die NS-Zeit (1938-1945)}

Der „Anschluss“ hatte auf die Wiener Gerichtsorganisation keine unmittelbaren Auswirkungen, ${ }^{108}$ sieht man davon ab, dass das Justizministerium in Wien nun sozusagen als "Filialbetrieb" geführt wurde: Reichsjustizministerium, Abteilung Österreich.

Im August 1938 wurden die reichsdeutschen Bezeichnungen „Amtsgericht” bzw. „Landgericht" eingeführt. ${ }^{109}$ Mit 15. Oktober 1938 wurde Wien wesentlich erweitert und in 26 Bezirke eingeteilt. ${ }^{110}$ "Neu“ waren die Bezirke 22 (GroßEnzersdorf, auch unter Einbeziehung von Teilen des bisherigen 21. Bezirkes), 23 (Schwechat),

\footnotetext{
104 Justizamtsblatt 1932, Nr. 2.

${ }^{105}$ BGBl. 76/1926; das sind nichtrichterliche Beamte, denen bestimmte Angelegenheiten der Gerichtsbarkeit zur selbständigen Besorgung übertragen werden können.

${ }^{106}$ BGBl. 222, 412/1929.

107 1944: Allgemeine Verfügung vom 19. September, Deutsche Justiz 249 (organisationsnormen, die wir nach heutigem Verständnis als Gesetz oder Verordnung im Reichsgesetzblatt suchen würden, ergingen auch mit "Allgemeinen Verfügungen" $[\mathrm{AV}]$, die in der amtlichen Zeitschrift [mit Amtsblattteil] „Deutsche Justiz" [DJ] veröffentlicht wurden); sodann BGBl. 196/1947; BGBl. 182, 184/1950; 1962 wurde mit Art. 87a B-VG eine verfassungsrechtliche Grundlage geschaffen (BGBl. 162), dazu erging das Rechtspflegergesetz, BGBl. 180/1962, abgelöst durch das Rechtspflegergesetz BGB1. 560/1985.

108 Wohl aber personelle.

109 dRGBl. I 998/1938, GB1Ö. 350/1938.

110 VOBl. Wien 23.
}

24 (Mödling), 25 (Liesing) und 26 (Klosterneuburg). ${ }^{111}$

Größere Änderungen gab es im Jahr 1939:

Das Reichsjustizministerium, Abteilung Österreich, wurde mit Ende Februar aufgelöst; ein Teil des Personals kam nach Berlin, Personal und Möbel wurden aber auch verwendet, um das neue Oberlandesgericht Linz zu errichten (mit 1. März 1939).112

Mit dem 1. April 1939 wurden der Oberste Gerichtshof und die Generalprokuratur aufgehoben und deren Agenden grundsätzlich dem Reichsgericht und der Reichsanwaltschaft übertragen. ${ }^{113}$

Mit 1. Mai 1939 wurden die fünf Wiener Gerichtshöfe zum Landgericht Wien vereinigt, weiters wurde das Amtsgericht (AG) Innere Stadt in AG Wien umbenannt ${ }^{114}$ und es übernahm die Agenden des StrafAG I und des Exekutionsgerichtes; das AG für Handelssachen wurde aufgelöst. ${ }^{115}$

Zum 1. Jänner 1940 wurden in Wien die AGe Leopoldstadt, Landstraße, Margareten und Josefstadt mit dem AGWien, sowie das AG Meidling mit dem AG Fünfhaus vereinigt, ${ }^{116}$ auch wurden die Zuständigkeiten des AG Wien

\footnotetext{
${ }^{111}$ Auch ansonsten kam es bei den Bezirken zu Grenzänderungen. Besonders ist hervorzuheben, dass die bisherigen Bezirke 14 (Rudolfsheim) und 15 (Fünfhaus) zum neuen 15. Bezirk vereinigt und der bisherige 13. Bezirk in die neuen Bezirke 13 (Hietzing) und 14 (Penzing) geteilt wurde.

112 dRGBl. I 166/1939; GB1Ö. 198/1939.

113 dRGB1. I 358, 448/1939; GB1Ö. 307, 353/1939; beim Reichsgericht wurden zwei Senate gebildet, die mit österreichischen Agenden betraut waren. In Österreich wurden den Oberlandesgerichten gewisse drittinstanzliche Agenden (bei Rekursen) übertragen.

${ }^{114}$ Die Bezeichnung „Amtsgericht Wien“ ist missverständlich, weil es ja nur für einen Teil von Wien zuständig war. Es war im Februar 1939 vom Justizpalast in das Gerichtsgebäude Riemergasse übersiedelt. 115 dRGBl. I 751/1939 = GBlÖ. 522/1939, abgeändert durch die AV DJ 699.

116 dRGBI. I 2439/1940.
} 
erweitert. ${ }^{117}$ Hervorzuheben ist, dass mit 1. März 1940 die Zuständigkeit zur Führung der Grundbücher, soweit die Landgerichte zuständig gewesen waren, auf die Amtsgerichte am Sitz des Gerichtshofes überging (in Wien auf das AG Wien). ${ }^{118}$ In der Folge gab es weitere auch kriegsbedingte Änderungen, die kaum nachhaltig waren. ${ }^{119}$

\section{Die Zweite Republik (seit 1945)}

Anders als im November 1918 musste im April 1945 die Justiz in Wien geradezu bei „null“ beginnen: Gebäude waren zerstört oder beschädigt, der Gerichtsbetrieb war eingestellt, es mangelte an Personal, das Justizressort war neu zu errichten und es waren die Einrichtungen des Deutschen Reiches geordnet überzuleiten. Noch Ende April 1945 wurde das Landgericht Wien wieder in - diesmal aber nur vier - selbständige Gerichtshöfe "zerlegt", nämlich in das Landesgericht für Zivilrechtssachen, das Handelsgericht, das Landesgericht für Strafsachen und den Jugendgerichthof (es gibt seither, anders als von 1920 bis 1938/39, nur ein Landesgericht für Strafsachen und nicht deren zwei). Die Gerichte erhielten wieder ihre früheren Bezeichnungen; aus dem BG Innere Stadt (AG Wien) wurde das Strafbezirksgericht ausgeschieden, das mit dem Landesgericht für Strafsachen am 18. Juni 1945 seinen Betrieb aufnahm. ${ }^{120}$ Vieles geschah damals zunächst eher „auf kurzem Weg“, die ge-

\footnotetext{
117 Weiters Anpassungen der AG-Sprengel in Wien; von den Burgenländischen Amtsgerichten verblieb nur Neusiedl bei Wien, die übrigen kamen zum LG Wr. Neustadt bzw. zum LG Graz. Überdies erfolgten mit der AV DJ 1899 u.a. Ausweitungen der Zuständigkeiten des AG Wien.

118 dRGBl I 301/1940; das Grundbuchs- und Landtafelamt des LGWien wurde demnach dem AGWien angegliedert, verblieb aber bis 1987 im Justizpalast. ${ }^{119}$ Siehe bei WALDSTÄTTEN, Gerichte, insbesondere 273-279.

120 Siehe WALDSTÄTtEN, Gerichte, 296-302, m.w.N.
}

setzlichen Grundlagen wurden zum Teil erst "nachgeschoben", 121 so insbesondere das Gerichtsorganisationsgesetz 1945,122 das Behördenüberleitungsgesetz, ${ }^{123}$ die Verordnungen über die Zuständigkeiten der Bezirksgerichte und der Gerichtshöfe erster Instanz in Wien. ${ }^{124}$ Die Zivilgerichte nahmen ihre judizielle Tätigkeit erst im Zuge des Sommers 1945 auf. ${ }^{125}$

Mit 1. Jänner 1955 wurden aus dem BG Innere Stadt Wien das BG für Handelssachen und das Exekutionsgericht (wieder-)errichtet. ${ }^{126}$

Es folgte nun hinsichtlich der Wiener Gerichtsorganisation eine längere Phase der Stabilität. Wenngleich nicht unmittelbar für die Gerichtsorganisation, so doch aus praktischer Sicht besonders relevant waren in der Folge die allmähliche Einführung der EDV im Gerichtsbetrieb und die Umstellung des Grundbuches auf EDV,127 dann die Umstellung des Handelsregisters auf das Firmenbuch. ${ }^{128}$

Auf Gerichtshofebene kam es mit 1. Jänner 1987 zur Errichtung des Arbeits- und Sozialgerichtes (für den Umfang des Sprengels des Landesgerichtes für ZRS Wien). ${ }^{129} 1993$ erhielten auch die bisherigen Kreisgerichte die Bezeichnung „Landesgericht", ${ }^{130}$ weiters wurden mit 1. Jänner 1997 die „Niederösterreichischen Umland-Bezirksgerichte Wiens" (Purkersdorf, Bruck an der Leitha , Groß-Enzersdorf, Hainburg, Kloster-

${ }^{121}$ Die Anfangsphase ist durch viel Improvisation gekennzeichnet.

122 StGBl. 47/1945.

123 StGBl. 94/1945.

124 StGBl. 122, 203/1945.

${ }^{125}$ Es herrschte "Stillstand der Rechtspflege“, die BGe entfalteten allenfalls eine beratende Tätigkeit oder auch in Außerstreit- und Grundbuchssachen.

${ }^{126}$ BGBl. 200/1954.

127 BGBl. 550/1980.

${ }^{128}$ BGBl. 10/1991.

${ }^{129}$ BGBl. 104/1985; das Arbeits- und Sozialgericht trat an die Stelle des Arbeitsgerichtes und der Schiedsgerichte der Sozialversicherung, soweit sie für den Sprengel des LG für ZRS zuständig waren.

${ }^{130}$ BGBl. 91/1993. 
neuburg und Schwechat sowie Mödling) den niederösterreichischen Landesgerichten St. Pölten, Korneuburg und Wr. Neustadt zugewiesen, womit die Zuständigkeit der Gerichtshöfe erster Instanz in Wien auf das Stadtgebiet beschränkt wurde. Schließlich wurde der Jugendgerichtshof mit Ende Juni 2003 aufgelöst, seine Agenden gingen teils auf das Landesgericht für Strafsachen und teils auf die Wiener Bezirksgerichte über. ${ }^{131}$

Auf bezirksgerichtlicher Ebene setzte in den 1980er-Jahren eine Entwicklung mit dem Ziel ein, „Vollgerichte“ unter Auflassung von Spezialbezirksgerichten (nämlich des StrafBG und des Exekutionsgerichtes) $\mathrm{zu}$ schaffen (das BG für Handelssachen blieb davon ausgenommen). ${ }^{132}$ Dazu erging das Bezirksgerichtsorganisationsgesetz für Wien,133 mit dem auch das neue BG Donaustadt zum 1. Jänner 1985 errichtet wurde. Die weiteren "Vollgerichte“ waren das BG Hernals per 1. Jänner 1989,134 dann das BG Döbling mit 1. Jänner 1991,135 das mit 1. Jänner 1993 wiedererrichtete BG Josefstadt, ${ }^{136}$ sodann mit 1. April 1997 das BG Hietzing, das BG Fünfhaus und das wiedererrichtete BG Meidling, womit zugleich das StrafBG und das Exekutionsgericht (nämlich das, was davon noch vorhanden war) aufgelöst wurden. ${ }^{137}$ Schließlich wurde noch mit 1. Jänner 2001 das BG Leopoldstadt wiedererrichtet. ${ }^{138}$

Zum 1. Juli 2016 sollten die Sprengel der Wiener Gerichtshöfe erster Instanz auf den Sprengel des BG Purkersdorf ausgedehnt, dieses BG aufgelöst

\footnotetext{
${ }^{131}$ BGB1. I 30/2003.

132 Ebenfalls ausgenommen waren die bezirksgerichtlichen Agenden des Jugendgerichtshofes, das erfolgte sodann mit der Auflösung des Jugendgerichtshofes im Jahr 2003.

133 BGB1. 203/1985.

${ }^{134}$ BGBl. 291/1988.

${ }^{135}$ BGB1. 260/1990.

${ }^{136}$ BGBl. 756/1992.

${ }^{137}$ BGBl. 761/1996.

${ }^{138}$ BGB1. I 57/1999.
}

und dessen Agenden dem BG Hietzing sowie dem BG für Handelssachen Wien zugewiesen werden, was aber durch das Gesetz BGBl. I 28/2016 i.V.m. der Bezirksgerichte-Verordnung Niederösterreich 2016 sozusagen "abgeblasen“ wurde. ${ }^{139}$

\footnotetext{
${ }^{139}$ Die Genesis dieser vorgesehenen Maßnahme war kompliziert: Für Wien bedarf es eines Gesetzes, für Niederösterreich einer Verordnung. Dies erfolgte zunächst mit der "Gerichtsorganisationsnovelle Wien-Niederösterreich“", BGB1. I 81/2012, in Verbindung mit der Bezirksgerichte-Verordnung Niederösterreich 2012, BGB1. II 204. Diese Organisationsänderung, die (zunächst) mit 1. 7. 2014 in Kraft treten sollte, war aber wohl wegen Verletzung der im Verfassungsrang stehenden Bestimmung des $\S 8$ Abs. 5 lit. d erster Halbsatz des Übergangsgesetzes (ÜG 1920) vom 1. 10. 1920 i.d.F. BGBl 368/1925 (evident) verfassungswidrig (S. dazu WALDSTÄTTEN, verfassungswidrige neue Organisationsvorschrift: Verletzung des "Schneideverbotes" - zu ähnlichen Maßnahmen in Oberösterreich siehe u.a. das Erkenntnis des VfGH vom 11.3. 2014, V 4/2014, und weitere Zahlen). Daraufhin wurde mit den Novellen BGBl. I 40/2014 (Art. 10) und BGBl. II 147/2014 (verkürzt gesagt) das Inkrafttreten auf den 1.7. 2016 verschoben und es wurde sodann das Hindernis „wegnovelliert" (Entfall des $\S 8$ Abs. 5 lit. d erster Halbsatz ÜG 1920: BGBl. I 77/2014). Dagegen gab es aber weiterhin verfassungsrechtliche Bedenken (siehe dazu auch HERBST, Gerichtsbezirk), sodass mit dem Gesetz BGB1. I 28/2016 die Novelle BGB1. I 81/2012 aufgehoben wurde (komplementär dazu erging die Bezirksgerichte-Verordnung Niederösterreich 2016, BGBl. II 147).
} 


\section{Korrespondenz:}

Dr. Alfred Waldstätten

Senatspräsident des VwGH

Wickenburggasse 19/14

1080 Wien

\section{Abkürzungen: \\ AG Amtsgericht \\ AV Allgemeine Verfügung \\ DJ Deutsche Justiz (Zeitschrift)}

dRGB1. Deutsches Reichsgesetzblatt

Siehe auch das allgemeine Abkürzungsverzeichnis: [http://www.rechtsgeschichte.at/files/abk.pdf]

\section{Literatur:}

Florian HERBST, Was ist ein Gerichtsbezirk?, in: JB1. 137 (2015) 545-556.

Josef KASERER, Handbuch der österreichischen Justizverwaltung. 4 Bde. (Wien 1882-1884).

Gerald KOHL, Die Anfänge der modernen Gerichtsorganisation in Niederösterreich (= Studien und Forschungen aus dem Niederösterreichischen Institut für Landeskunde 83, St. Pölten 2000).

Ferdinand OPLL, Wien im Bild historischer Karten. Die Entwicklung der Stadt bis in die Mitte des 19. Jahrhunderts (Wien-Köln-Graz 22004).

Alfred WALDSTÄTtEN, Staatliche Gerichte in Wien seit Maria Theresia. Beiträge zu ihrer Geschichte. Ein Handbuch (= Forschungen und Beiträge zur Wiener Stadtgeschichte 54, Innsbruck-Wien-Bozen 2011).

Alfred WALDSTÄTtEN, Eine verfassungswidrige neue Organisationsvorschrift?, in: RZ 92 (2014) 28-29. 